\title{
Basic Principles of Atomic Force Microscopy for Life Sciences
}

\author{
Helen A. McNally
}

Department of Electrical and Computer Engineering Technology and The Birck Nanotechnology Center, Purdue University, West Lafayette, IN 47907

Scanning Probe Microscopy (SPM) is a generalize name for a series of microscopy techniques involving a scarp probe interacting with a sample of interest. The interactions examined typically give rise to the second term in the name. For example, Atomic Force Microscopy (AFM) investigates long and short range atomic forces between the sample and probe; whereas Magnetic Force Microscopy examines magnetic interactions. AFM was first introduced in 1986 [1] which eliminated the requirement of a conducting sample from previous SPM systems. Since then the AFM has quickly become an instrument of choice for life scientists. A second development extended the capabilities of AFM to fluids [2] allowing investigations in physically relevant conditions. And yet, AFM is a relatively immature technique which is currently being improved upon, modified or adapted to newly developed techniques. This paper will introduce the AFM capabilities (or limitations) for life scientists.

AFM can be used to image the surface topography, measure material characteristics and interact with a sample of interest. This can be done at physiologically relevant conditions or changed as necessary to emulate atypical conditions such as varied temperatures, changing gases/liquids, introduction of chemistry, and even now hyperbaric conditions [3.] Various modes of AFM are used to obtain the data desired. These include static and dynamic imaging modes and force measurements. A variety of adaptations have been made to AFMs specifically for the life sciences. Entire systems have been integrated with optical microscopes, heated stages have been included and flow-through systems now allow a continuous bathing of the sample in the appropriate solution.

To date, the author has used the AFM to provide unparalleled resolution of living neural cells [4], measure the cells' reaction to changing temperatures [5] and the introduction of endotoxins [6], as well as to emulate the traumatic event of spinal cord injury at the cellular level [7.] The images in Fig. 1 show a living neural cell growth cone as it extends across the AFM imaging area. The entire growth cone can be investigated or the fine fillipodia can be investigated at much smaller resolution. Fig. 2 shows a typical force curve on the neural cell body. This data can be used to investigate the viscosity of the cellular membrane and ultimately the health of the cell.

Proteins, Viruses and Macromolecules are also being investigated by AFM. Atomic resolution is possible (under the correct conditions.) Protein interactions can be measured by modifying the probe. Dynamic processes are being viewed. The possibilities are virtually limitless. AFM has tremendous potential in the life sciences.

References

[1] Binnig, G., Quate, C.F., \& Gerber, Ch., Atomic Force Microscopy, Physics Review Letts, V. 56, N. 9, pp. 930-933., 1986. 
[2] Hansma, P.K., Cleveland, J.P., Radmacher, M., Walters, D.A., Hillner, P.E., Bezanilla, M., Prater, C.B., Massie, J., Fukunaga, L., Gurley, J., and Elings, V., Tapping mode atomic force microscopy in liquids, Appl. Phys. Lett, V. 64, N. 13, pp. 1738-1740, 1994.

[3] McNally, H.A. \& D’Agostino, D. (2010). Hyperbaric Atomic Force Microscopy, Imaging and Microscopy,

[4] McNally, H.A., Rajwa, B., Sturgis, J., \& Robinson, J.P., (2005). Comparative threedimensional imaging of living neuron morphology imaged with confocal and atomic force microcopy. Journal of Neuroscience Methods, V. 142, pp.177-184.

[5] Mustata, M., Ritchie, K., \& McNally, H.A., (2010). Neuronal Elasticity as Measured by Atomic Force Microscopy. Journal of Neuroscience Methods, V. 186, pp.35-41.

[6] Liu-Snyder, P., McNally, H.A., Shi, R., \& Borgens, R., (2006). Acrolein-Mediated Mechanisms of Neuronal Death. Journal of Neuroscience Research, V.84, pp.209-218.

[7] McNally H.A., \& Borgens, R., (2004). Three-Dimensional Form in Living and Dying Neurons with Atomic Force Microscopy. Journal of Neurocytology, V.33, I.2, pp. 251-258.

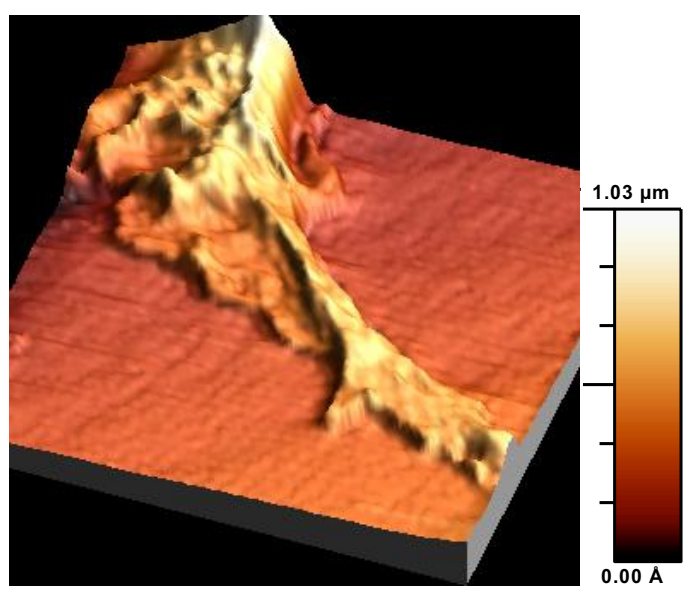

FIG. 1. A neural cell growth cone, image is $20 \mathrm{X} 20$ microns, the color bar denoted the $\mathrm{Z}$ scale.

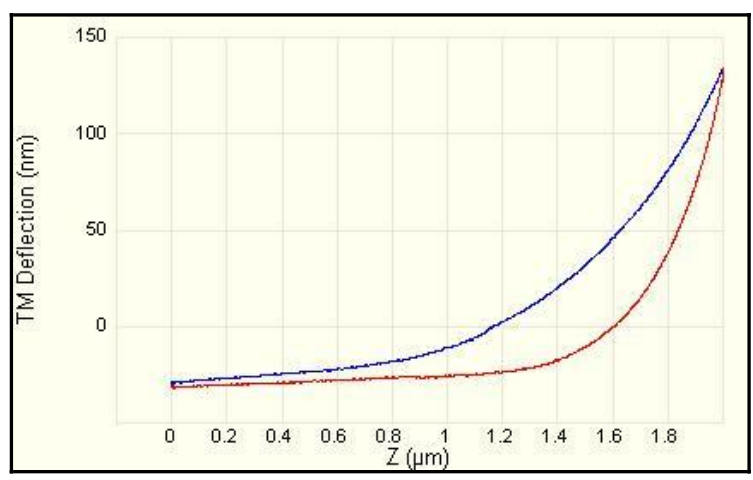

FIG. 2. AFM Force Curve on a Neural Cell Body, The red trace represents the extending portion of the force curve while the blue curve represents the retraction portion. 